\title{
HLA-DR, HLA-DQB1 and PTPN22 gene polymorphism: association with age at onset for autoimmune diabetes
}

Anna Okruszko, Barbara Szepietowska, Natalia Wawrusiewicz-Kurylonek, Maria Górska, Adam Krętowski, Małgorzata Szelachowska

Department of Endocrinology, Diabetology and Internal Medicine, Medical University of Bialystok, Poland

Submitted: 30 May 2012

Accepted: 20 August 2012

Arch Med Sci 2012; 8, 5: 874-878

DOI: 10.5114/aoms.2012.31619

Copyright @ 2012 Termedia \& Banach

\section{Abstract}

Introduction: Autoimmune diabetes has different clinical manifestations related to the age at onset. It is divided into several subtypes, including "classical" type 1 diabetes (T1D) and latent autoimmune diabetes in adults (LADA). The LADA is considered a slowly progressing subtype of autoimmune diabetes, although the clinical picture is more similar to type 2 diabetes.

Material and methods: The aim of this study is to investigate whether genetic predisposition influences age at onset in autoimmune diabetes. We studied rs2476601 PTPN22 gene polymorphism and HLA DR, HLA-DQB1 in 175 patients with classical type 1 diabetes, 80 LADA, and 151 control subjects from northeast Poland.

Results: The frequencies of the PTPN22 TT genotype were higher in the group of patients with classical type 1 diabetes $(6.3 \%)$ and LADA $(11.3 \%)$ than in control subjects $(0.7 \%)(p=0.02$ and $p=0007$, respectively). In patients with classical type 1 diabetes we observed an increasing trend in frequencies of genotype TT dependent on age at onset (3.9\% (0-5 year olds), 6.0\% (6-15 year-olds), $8.2 \%$ (16-25 year olds), $p=0.048$ ). The incidence of predisposing human leukocyte antigen (HLA) genotypes HLA DR3/DQB1*02 and $D R 4 / D Q B 1^{*} 0302$ was found to decrease in the group with type 1 diabetes in relation to age at onset and LADA (HLA DR3/DQB1*02 - 69.2\% (0-5 year olds), 57.0\% (6-15 year olds), 51.0\% (16-25 year olds), $46.3 \%$ (LADA), $p=0.032$; HLA DR4/DQB1*0302 $-80.8 \%$ (0-5 year olds), $63.0 \%$ (6-15 year olds), 51.0\% (16-25 year olds), $43.8 \%$ (LADA), $p=0.0003)$, and to increase for the protective allele DQB1*0602 (0.0\% (0-5 year olds), $1.0 \%$ (6-15 year olds), $2.0 \%$ (16-25 year olds), $6.3 \%$ (LADA), $p=0.029$ ).

Conclusions: Thus, age at onset for autoimmune diabetes appears to be related to a combination of predisposing and protective HLA alleles. Against a background of HLA genetic predisposition, other non-HLA loci may influence age at onset for late autoimmune diabetes.

Key words: type 1 diabetes, latent autoimmune diabetes in adults, PTPN22, HLA.

\section{Introduction}

There are two major clinical manifestations of autoimmune diabetes: type 1 diabetes (T1D) and latent autoimmune diabetes in adults (LADA) $[1,2]$. The LADA is characterized by adult onset, no requirement for insulin for several months following diagnosis and presence of islet autoantibodies similar to those found in type 1 diabetes, but with a clinical picture more similar to type 2 diabetes [3].

\author{
Corresponding author: \\ Małgorzata Szelachowska MD \\ Department of Endocrinology, \\ Diabetology and Internal \\ Medicine \\ Medical University \\ of Bialystok \\ 24 A M. Skłodowskiej-Curie St \\ 15-276 Białystok, Poland \\ Phone: +48 857468607 , \\ +48 857468239 \\ Fax: +48 857447611 \\ E-mail: \\ mszelachowska@poczta.onet.pl
}


Patients with LADA have been found to share similar genetic susceptibility traits with type 1 diabetics. These include (i) protein tyrosine phosphatase non-receptor type 22 (PTPN22) gene polymorphism [4, 5], (ii) insulin gene variable tandem repeats (INS VNTR) [6] and (iii) HLA locus [7] with more common protective and less frequent predisposing HLA genotypes in LADA than in T1D $[5,8]$. Prevalence of LADA and classification criteria have been previously described in the Polish population [9].

Although the role of PTPN22 in predisposition to autoimmune diabetes has previously been described [10], its role in modifying age at onset in autoimmune diabetes is not fully understood and observations reported to date are contradictory [11-15]. Therefore, the aim of this study was to evaluate the correlation between genetic predisposition - in terms of PTPN22 polymorphism and HLA genotypes - and age at onset of autoimmune diabetes in two patient populations with different disease manifestations (T1D and LADA).

\section{Material and methods}

The study protocol was approved by the Ethics Committee of the Medical Academy in Bialystok. Informed consent was obtained from all patients before blood sampling. Three groups of individuals from the Bialystok region of Poland were included: 1) 175 patients with type 1 diabetes selected from the register of new cases in the Bialystok region, which was established in 1994 as part of the EURODIAB TIGER program. Diagnosis of T1D was made according to the criteria defined by the World Health Organization in 1985: presence of ketosis, low body mass index (BMI), and requirement for insulin therapy.

2) 80 LADA patients. Diabetes was diagnosed according to the World Health Organization (WHO) 1999 criteria. Classification for LADA: age at onset $>30$ years, positive GAD and/or IAA and/or IA2 autoantibodies, no requirement for insulin treatment for 3-6 months from diagnosis.

3) The control group consisted of a sample of 151 unrelated healthy volunteer subjects from the medical staff of our hospital and medical students living in the Bialystok region, who had no family history of diabetes or other autoimmune diseases.

GADA, IA2 and IAA were measured using commercially available RIA kits (CIS Bio International, France).

\section{Single nucleotide polymorphism genotyping}

DNA was extracted from peripheral blood leukocytes. Genotyping of the rs2476601 polymorphism of PTPN22 was performed using PCR sequencespecific primers with the $A B I$ Prism 310 genetic analyzer (Applied Biosystems, Stanford, TX). Primer sequences for position -185 were forward 5'-tcaccagcttcctcaaccaca-3' and reverse 5'-gataatgttgcttcaacggaattt-3'. HLA genotyping was performed as previously described [16].

\section{Statistical analysis}

The genotype distribution differences in the population studied were assessed with Haploview v3.2 (http://www.broad.mit.edu/mpg/haploview).

The $\chi^{2}$ test or Fisher's exact probability test were used to estimate the differences in the distribution of alleles, genotypes, and haplotypes between the studied groups. Trend analysis was performed with the Cochran-Armitage test (SAS/STAT version 9.0 SAS Institute).

Values of $p$ were corrected for the number of different haplotypes tested $(P C)$. Statistical significance was defined as $p<0.05$.

\section{Results}

Clinical characteristics of the subjects are presented in Table I. Mean age at diagnosis ( \pm standard deviation (SD)) was $20.9 \pm 12.5$ years for type 1 and $45.4 \pm 9.6$ years for LADA.

\section{PTPN22 in patients with T1D and LADA}

All genotypes of the PTPN22 1858T variant were in Hardy-Weinberg equilibrium. Frequencies of allele $\mathrm{T}$ and distribution of genotypes CC, CT and TT were similar in patients with T1D and LADA (1858T: $18.9 \%$ (T1D) vs. $23.8 \%$ (LADA), $\chi^{2}=1.62, p=0.20$ and CC/CT/TT: $68.6 \% / 25.1 \% / 6.3 \%$ (T1D) vs. $63.7 \% /$ $25.0 \% / 11.3 \%$ (LADA), $\chi^{2}=1.91, p=0.38$.

The frequencies of the PTPN22 allele T and genotype TT were higher in the group of patients with T1D than in the controls ( $18.9 \%$ vs. $12.6 \%, p=0.03$; $6.3 \%$ vs. $0.7 \%, p=0.02$, respectively). Data are presented in Table II. The frequencies of the PTPN22 allele $\mathrm{T}$ and genotype TT in the group of patients with LADA were also higher than in controls $(23.8 \%$ vs. $12.6 \%, p=0.002 ; 11.3 \%$ vs. $0.7 \%, p=0.0007$, respectively) (Table II).

\section{Association of PTPN22 with age at onset for T1D}

The group of 175 patients with type 1 diabetes was divided according to age of diabetes onset:

Table I. Clinical characteristics of subjects

\begin{tabular}{|lccc|}
\hline Parameter & $n(\mathrm{M} / \mathrm{F})$ & $\begin{array}{c}\text { Mean age } \\
\text { [years] }\end{array}$ & $\begin{array}{c}\text { Mean age } \\
\text { at diagnosis } \\
\text { [years] }\end{array}$ \\
\hline T1D & $175(89 / 86)$ & $20.9 \pm 12.5$ & $14.3 \pm 9.1$ \\
\hline LADA & $80(42 / 38)$ & $45.4 \pm 9.6$ & $43.2 \pm 9.1$ \\
\hline
\end{tabular}

Data are expressed as mean $\pm S D$ 
group I - diabetes onset at 0-5 years of age; group II - 6-15 years; and group III - 16-25 years.

The frequencies of allele $\mathrm{T}$ and genotype TT increased proportionately to age at diagnosis of type 1 diabetes. Allele T: 13.5\% (0-5 years), 17.2\% (6-15 years), $25 \%$ (16-25 years), $p=0.017$; genotype TT: 3.9\% (0-5 years), 6.0\% (6-15 years), 8.2\% (16-25 years), $p=0.048$ (Table III)

\section{Association of HLA with age at onset of autoimmune diabetes (type 1 and LADA)}

We investigated the association between the HLA genotypes DR3/DQB1*02 and DR4/DQB1*0302 and increasing risk for T1D, as well as the association between the HLA genotype DQB1*0602 and decreasing risk for T1D in T1D patients (divided into 3 groups according to age at onset) and in LADA patients. As age at onset increased, we observed decreasing frequencies of the predisposing HLA alleles HLA DR3/DQB1*02 (69.2\% (T1D 0-5 years),
$57.0 \%$ (T1D 6-15 years), 51.0\% (T1D 16-25 years), $46.3 \%$ (LADA), $p=0.032$ ) and DR4/DQB1*0302 (80.8\% (T1D 0-5 years), 63.0\% (T1D 6-15 years), $51.0 \%$ (T1D 16-25 years), 43.8\% (LADA), $p=0.0003$ ) and an increased incidence of the protective allele DQB1*0602 (0.0\% (T1D 0-5 years), 1.0\% (T1D 6-15 years), $2.0 \%$ (T1D 16-25 years), 6.3\% (LADA), $p=0.029$ ) (Table IV).

\section{Discussion}

Diagnosis of LADA is based on autoimmune status and age at onset [17]. The role that genetic predisposition may play in modifying age at diabetes onset and clinical presentation of autoimmune diabetes is unclear. Previous observations in review articles $[18,19]$ and recent analysis of siblings affected and not affected by type 1 diabetes [20] showed that age of manifestation of symptoms in T1D is strongly related to HLA genetic predisposition. In our study we have shown that age at onset for

Table II. Frequency of alleles and genotypes of 1858 PTPN22 gene in controls, T1D and LADA

\begin{tabular}{|lccc|}
\hline Allele/genotype PTPN22 1858 & Controls $(n=151)$ & Type 1 diabetes $(n=175)$ & LADA $(n=80)$ \\
\hline C & $264(87.4 \%)$ & $284(81.1 \%)$ & $122(76.2 \%)$ \\
\hline T & $38(12.6 \%)$ & $66(18.9 \%)$ & $38(23.8 \%)$ \\
\hline T1D vs. controls: $\chi^{2}=4.76 ; p=0.029$, LADA vs. controls: $\chi^{2}=9.5 ; p=0.002$ \\
\hline CC & $114(75.5)$ & $120(68.6 \%)$ & $51(63.7 \%)$ \\
\hline CT & $36(23.8 \%)$ & $44(25.1 \%)$ & $20(25.0 \%)$ \\
\hline TT & $1(0.7 \%)$ & $11(6.3 \%)$ & $9(11.3 \%)$ \\
\hline T1D vs. controls: $\chi^{2}=7.6 ; p=0.023$, LADA vs. controls: $\chi^{2}=14.6 ; p=0.0007$ & \\
\hline
\end{tabular}

Table III. Frequency of alleles and genotypes of 1858 PTPN22 gene in patients with T1D relating to age at onset

\begin{tabular}{|lcccc|}
\hline $\begin{array}{l}\text { Allele/genotype } \\
\text { PTPN22 1858 }\end{array}$ & $\begin{array}{c}\text { Type 1 diabetes age } \\
\text { range 0-5 years }(n=26)\end{array}$ & $\begin{array}{c}\text { Type } 1 \text { diabetes age } \\
\text { range 6-15 years }(n=100)\end{array}$ & $\begin{array}{c}\text { Type } 1 \text { diabetes age } \\
\text { range 16-25 years }(n=49)\end{array}$ & $\begin{array}{c}\text { Value of } p \\
\text { for trend }\end{array}$ \\
\hline C C & $45(86 \%)$ & $164(82.8 \%)$ & $63(71.6 \%)$ & \multirow{2}{*}{$0.017^{\star}$} \\
\hline T & $7(13.5 \%)$ & $34(17.2 \%)$ & $25(28.4 \%)$ & \\
\hline CC & $20(76.9 \%)$ & $72(72.0 \%)$ & $28(57.1 \%)$ & \multirow{2}{*}{$0.048^{*}$} \\
\cline { 1 - 3 } CT & $5(19.2 \%)$ & $22(22.0 \%)$ & $17(34.7 \%)$ & \\
\hline TT & $1(3.9 \%)$ & $6(6.0 \%)$ & $4(8.2 \%)$ & \\
\hline
\end{tabular}

${ }^{*}$ Cochran-Armitage trend test

Table IV. Frequency of HLA DR3/DQB1*02, DR4/DQB1*0302, DQB1*0602 genotypes relating to age at onset of T1D and LADA

\begin{tabular}{|lccccc|}
\hline Genotype HLA & $\begin{array}{c}\text { Type } 1 \text { diabetes } \\
\text { age range } \\
0-5 \text { years }(n=26)\end{array}$ & $\begin{array}{c}\text { Type } 1 \text { diabetes } \\
\text { age range } \\
\text { 6-15 years }(n=100)\end{array}$ & $\begin{array}{c}\text { Type } 1 \text { diabetes } \\
\text { age range } \\
16-25 \text { years }(n=49)\end{array}$ & $\begin{array}{c}\text { LADA }>30 \\
(n=80)\end{array}$ & $\begin{array}{c}\text { Value of } p \\
\text { for trend }\end{array}$ \\
\hline DR3/DQB1*02 & $18(69.2 \%)$ & $57(57.0 \%)$ & $25(51.0 \%)$ & $37(46.3 \%)$ & 0.032 \\
\hline DR4/DQB1*0302 & $21(80.8 \%)$ & $63(63.0 \%)$ & $25(51.0 \%)$ & $35(43.8 \%)$ & 0.0003 \\
\hline DQB1 ${ }^{*} 0602$ & $0(0.0 \%)$ & $1(1.0 \%)$ & $1(2.0 \%)$ & $5(6.3 \%)$ & 0.029 \\
\hline
\end{tabular}

${ }^{*}$ Cochran-Armitage trend test 
autoimmune diabetes is related to an inverse combination of predisposing and protective HLA alleles. We observed decreasing frequencies with increasing age at onset of the high-risk alleles HLA $D R 3 / D Q B 1^{\star} 02$ and $D R 4 / D Q B 1^{\star} 0302$ and increasing frequencies with increasing age at onset of the protective allele $D Q B 1^{\star} 0602$ in patients with type 1 diabetes and LADA. Previous studies [4, 5, 7, 21, 22] showed that patients with LADA had an increased frequency of $D Q B 1^{\star} 0602$, suggesting that the presence of "protective" HLA alleles may have a predominant role in delaying onset of autoimmune diabetes [22].

A predisposing role of PTPN22 gene polymorphism for type 1 diabetes [12, 13, 23, 24], and also for LADA, was previously described [4, 5]. However, the role of the PTPN22 gene in modifying age at onset for autoimmune diabetes was not clear. In a study conducted by Kordonouri et al. [11] carriers of genotype TT of the PTPN22 gene had an earlier onset of disease compared to patients with the CT and CC genotype, and a similar trend was observed by others $[12,13]$. Other studies investigated the role of a predisposing PTPN22 genotype in progression to type 1 diabetes [15] and loss of residual beta cell function [10]. A study performed in the Denver Diabetes Center, however, did not observe an association of the PTPN22 gene predisposing TT genotype with age at onset for patients with type 1 diabetes [14].

In the present study, the frequency of the PTPN22 gene high-risk genotype TT increased with increasing age at diagnosis for patients with T1D. In addition, the frequency of genotype TT in patients with LADA was twice that of patients with type 1 diabetes. Consistent with previous observations $[4,5,22]$ our study revealed the PTPN22 genotype TT to be related to an increased risk for LADA. This observation may suggest that significance of non-HLA related genetic predisposition, in this study PTPN22 genotype TT, may have a significant effect on the age at diagnosis for latent autoimmune diabetes and/or type 1 diabetes in young adults, whereas HLA-related genetic predisposition determined age at onset in children with type 1 diabetes.

In conclusion, age at onset for autoimmune diabetes is linked to a combination of predisposing and protective HLA alleles. Against a background of HLA genetic predisposition, other non-HLA loci may influence age at onset of late autoimmune diabetes and this association may be more significant than in patients with classic type 1 diabetes.

\section{References}

1. Gale EA. Latent autoimmune diabetes in adults: a guide for the perplexed. Diabetologia 2005; 48: 2195-9.
2. Palmer JP, Hampe CS, Chiu H, Goel A, Brooks-Worrell BM. Is latent autoimmune diabetes in adults distinct from type 1 diabetes or just type 1 diabetes at an older age? Diabetes 2005; 54 Suppl 2: S62-7.

3. Leslie RD, Kolb H, Schloot NC, et al. Diabetes classification: grey zones, sound and smoke: Action LADA 1. Diabetes Metab Res Rev 2008; 24: 511-9.

4. Petrone A, Suraci C, Capizzi M, et al. The protein tyrosine phosphatase nonreceptor 22 (PTPN22) is associated with high GAD antibody titer in latent autoimmune diabetes in adults: Non Insulin Requiring Autoimmune Diabetes (NIRAD) Study 3. Diabetes Care 2008; 31: 534-8.

5. Cervin C, Lyssenko V, Bakhtadze E, et al. Genetic similarities between latent autoimmune diabetes in adults, type 1 diabetes, and type 2 diabetes. Diabetes 2008; 57: 1433-7.

6. Desai M, Zeggini E, Horton VA, et al. The variable number of tandem repeats upstream of the insulin gene is a susceptibility locus for latent autoimmune diabetes in adults. Diabetes 2006; 55: 1890-4.

7. Desai M, Zeggini E, Horton VA, et al. An association analysis of the HLA gene region in latent autoimmune diabetes in adults. Diabetologia 2007; 50: 68-73.

8. Szepietowska B, Moczulski D, Wawrusiewicz-Kurylonek N, Grzeszczak W, Gorska M, Szelachowska M. Transcription factor 7-like 2-gene polymorphism is related to fasting $C$ peptide in latent autoimmune diabetes in adults (LADA). Acta Diabetol 2010; 47: 83-6.

9. Szepietowska B, Głębocka A, Puch U, Górska M, Szelachowska M. Latent autoimmune diabetes in adults in a population based cohort of Polish patients with newly diagnosed diabetes mellitus. Arch Med Sci 2012; 8: 491-5.

10. Petrone A, Spoletini M, Zampetti S, et al. The PTPN22 1858T gene variant in type 1 diabetes is associated with reduced residual beta-cell function and worse metabolic control. Diabetes Care 2008; 31: 1214-8.

11. Kordonouri O, Hartmann R, Badenhoop K, Kahles H, Ilonen J. PTPN22 1858T allele is associated with younger age at onset of type 1 diabetes and is not related to subsequent thyroid autoimmunity. Hum Immunol 2010; 71: 731-2.

12. Ladner MB, Bottini N, Valdes AM, Noble JA. Association of the single nucleotide polymorphism C1858T of the PTPN22 gene with type 1 diabetes. Hum Immunol 2005; 66: 60-4.

13. Fedetz M, Matesanz F, Caro-Maldonado A, et al. The 1858T PTPN22 gene variant contributes to a genetic risk of type 1 diabetes in a Ukrainian population. Tissue Antigens 2006; 67: 430-3.

14. Steck AK, Liu SY, McFann K, et al. Association of the PTPN22/LYP gene with type 1 diabetes. Pediatr Diabetes 2006; 7: 274-8.

15. Hermann R, Lipponen K, Kiviniemi M, et al. Lymphoid tyrosine phosphatase (LYP/PTPN22) Arg620Trp variant regulates insulin autoimmunity and progression to type 1 diabetes. Diabetologia 2006; 49: 1198-208.

16. Kretowski A, Kinalska I. DQA1 and DQB1 HLA genes as markers of insulin-dependent diabetes mellitus in the Polish population. Pol Arch Med Wewn 1999; 101: 205-11.

17. Leslie RD, Williams R, Pozzilli P. Type 1 diabetes and latent autoimmune diabetes in adults: one end of the rainbow. J Clin Endocrinol Metab 2006; 91: 1654-9.

18. Steck AK, Rewers MJ. Genetics of type 1 diabetes. Clin Chem 2010; 57: 176-85.

19. Dorman JS, Bunker CH. HLA-DQ locus of the human leukocyte antigen complex and type 1 diabetes mellitus: a HuGE review. Epidemiol Rev 2000; 22: 218-27. 
20. Morahan G, Mehta M, James I, et al. Tests for genetic interactions in type 1 diabetes: linkage and stratification analyses of 4,422 affected sib-pairs. Diabetes 2011; 60: 1030-40.

21. Tuomi T, Carlsson A, Li H, Isomaa B, et al. Clinical and genetic characteristics of type 2 diabetes with and without GAD antibodies. Diabetes 1999; 48: 150-7.

22. Andersen MK, Lundgren V, Turunen JA, et al. Latent autoimmune diabetes in adults differs genetically from classical type 1 diabetes diagnosed after the age of 35 years. Diabetes Care 2010; 33: 2062-4.

23. Kahles H, Ramos-Lopez E, Lange B, Zwermann O, Reincke M, Badenhoop K.Sex-specific association of PTPN22 1858T with type 1 diabetes but not with Hashimoto's thyroiditis or Addison's disease in the German population. Eur J Endocrinol 2005; 153: 895-9.

24. Fichna $M$, Zurawek $M$, Januszkiewicz-Lewandowska $D$, Fichna P, Nowak J. PTPN22, PDCD1 and CYP27B1 polymorphisms and susceptibility to type 1 diabetes in Polish patients. Int J Immunogenet 2010; 37: 367-72. 